\title{
Exercício físico como ferramenta para o envelhecimento ativo: Uma reflexão teórica
}

\author{
Physical exercise as a tool for active aging: A theoretical reflection \\ El ejercicio físico como herramienta para el envejecimiento activo: Una reflexión teórica
}

Recebido: 29/05/2021 | Revisado: 09/06/2021 | Aceito: 10/06/2021 | Publicado: 25/06/2021

\author{
Bruno Pereira do Prado \\ ORCID: https://orcid.org/0000-0002-0593-0237 \\ Faculdade Independente do Nordeste, Brasil \\ E-mail: jcarlospradobruno@gmail.com \\ Luciana Araújo dos Reis \\ ORCID: https://orcid.org/0000-0002-0867-8057 \\ Universidade Estadual do Sudoeste da Bahia, Brasil \\ E-mail: lucianauesb@yahoo.com.br \\ Wagner Couto Assis \\ ORCID: https://orcid.org/0000-0001-7802-2443 \\ Universidade Estadual do Sudoeste da Bahia, Brasil \\ E-mail: wagnerassis2010@hotmail.com \\ Flávia Rocha Brito \\ ORCID: https://orcid.org/ 0000-0002-7273-4957 \\ Faculdade Pitágoras de Medicina, Brasil \\ E-mail: flaviarrbrito@gmail.com \\ Lilian Almeida Nascimento Rabelo \\ ORCID: https://orcid.org/0000-0001-7457-6510 \\ Faculdade de Tecnologia e Ciências, Brasil \\ E-mail: lynascimento@hotmail.com \\ Frank Evilácio de Oliveira Guimarães \\ ORCID: https://orcid.org/0000-0003-4739-3676 \\ Universidade Católica do Salvador, Brasil \\ E-mail: frankevilacio@hotmail.com \\ Isnara Teixeira de Brito \\ ORCID: https://orcid.org/0000-0001-7916-2085 \\ Universidade Estadual do Sudoeste da Bahia, Brasil \\ E-mail: isnara.britto@hotmail.com \\ Luana Araújo dos Reis \\ ORCID: https://orcid.org/0000-0002-9263-083X \\ Faculdade Independente do Nordeste, Brasil \\ Instituto Multidisciplinar em Saúde, Brasil \\ E-mail: luareis1@hotmail.com
}

\begin{abstract}
Resumo
Objetivo: descrever a importância do exercício físico no envelhecimento ativo, a partir de uma reflexão teórica. Metodologia: Trata-se de um ensaio teórico de caráter reflexivo da literatura sobre a prática de exercício físico do idoso, por meio de uma revisão bibliográfica não sistemática sobre o tema "exercício físico e saúde da pessoa idosa", e também através da percepção dos autores acerca da temática. Como estratégia de busca foram definidas bases de dados eletrônicas, bibliotecas virtuais, respectivamente a Literatura Latino-Americana e do Caribe em Ciências da Saúde (LILACS), Medical Literature Analysis and Retrieval System Online (MEDLINE) e Scientific Eletronic Library Online (SCIELO), utilizando os descritores padronizados pelo Descritores em Ciências da Saúde (Decs): Exercício Físico; Saúde do Idoso; Envelhecimento Saudável, e seus correspondentes em inglês. O levantamento limitou-se aos artigos publicados em português, inglês e espanhol, publicados no período de 2010 a 2020, disponíveis na íntegra e cujos resultados abrangem o tema desta pesquisa. Resultado: A análise da literatura foi disposta em três subtemas: Nuances do envelhecimento; Envelhecimento ativo: caminhos para viver bem; e Exercício físico na terceira idade. Conclusão: O exercício físico impacta de forma positiva no envelhecimento ativo, contribuindo para a melhora nas capacidades cognitivas e funcionais, reduzindo o risco de doenças crônicas e incapacitantes, aumentando a qualidade de vida e bem-estar, além de ser responsável pela inserção da pessoa idosa nos ambientes sociais. No entanto, há lacunas quanto à execução segura para cada modalidade de exercício físico e os possíveis riscos à saúde relacionados a eventos adversos como lesão musculoesquelética, fadiga muscular e complicações cardiovasculares, uma vez que o desenvolvimento de tais práticas deve ser realizado de forma singular, atendendo a demanda individual de cada pessoa.
\end{abstract}

Palavras-chave: Exercício físico; Saúde do idoso; Envelhecimento saudável. 


\begin{abstract}
Objective: to describe the importance of physical exercise in active aging, from a theoretical reflection. Methodology: This is a theoretical essay of a reflective nature of the literature on the practice of physical exercise by the elderly, through a non-systematic bibliographic review on the theme "physical exercise and health of the elderly", and also through the perception of the authors about the theme. As a search strategy, electronic databases, virtual libraries were defined, respectively the Latin American and Caribbean Literature in Health Sciences (LILACS), Medical Literature Analysis and Retrieval System Online (MEDLINE) and Scientific Electronic Library Online (SCIELO), using the descriptors standardized by the Health Sciences Descriptors (Decs): Physical Exercise; Elderly Health; Healthy Aging, and its English counterparts. The survey was limited to articles published in Portuguese, English and Spanish, published in the period from 2010 to 2020, available in full and whose results cover the topic of this research. Result: The analysis of the literature was arranged in three sub-themes: Nuances of aging; Active aging: ways to live well; and Physical exercise in old age. Conclusion: Physical exercise positively impacts active aging, contributing to the improvement in cognitive and functional abilities, reducing the risk of chronic and disabling diseases, increasing quality of life and well-being, in addition to being responsible for the person's insertion elderly in social environments. However, there are gaps regarding the safe execution for each type of physical exercise and the possible health risks related to adverse events such as musculoskeletal injury, muscle fatigue and cardiovascular complications, since the development of such practices must be carried out in a unique way, meeting the individual demand of each person.
\end{abstract}

Keywords: Exercise; Health of the elderly; Healthy aging.

\title{
Resumen
}

Objetivo: describir la importancia del ejercicio físico en el envejecimiento activo, a partir de una reflexión teórica. Metodología: Se trata de un ensayo teórico de carácter reflexivo de la literatura sobre la práctica del ejercicio físico por parte del adulto mayor, a través de una revisión bibliográfica no sistemática sobre el tema "ejercicio físico y salud del adulto mayor", y también a través de la percepción de los autores sobre el tema. Como estrategia de búsqueda se definieron bases de datos electrónicas, bibliotecas virtuales, respectivamente Literatura Latinoamericana y Caribeña en Ciencias de la Salud (LILACS), Sistema de Análisis y Recuperación de Literatura Médica en Línea (MEDLINE) y Biblioteca Electrónica Científica en Línea (SCIELO), utilizando los descriptores estandarizados por los Descriptores de Ciencias de la Salud (Decs): Ejercicio Físico; Salud de los ancianos; Healthy Aging y sus homólogos ingleses. La encuesta se limitó a artículos publicados en portugués, inglés y español, publicados de 2010 a 2020, disponibles en su totalidad y cuyos resultados cubren el tema de esta investigación. Resultados: El análisis de la literatura se organizó en tres subtemas: Matices del envejecimiento; Envejecimiento activo: formas de vivir bien; y Ejercicio físico en la vejez. Conclusión: El ejercicio físico tiene un impacto positivo en el envejecimiento activo, contribuyendo a la mejora de las capacidades cognitivas y funcionales, reduciendo el riesgo de enfermedades crónicas e invalidantes, aumentando la calidad de vida y el bienestar, además de ser responsable de la inserción del persona anciana en entornos sociales. Sin embargo, existen lagunas en cuanto a la ejecución segura de cada tipo de ejercicio físico y los posibles riesgos para la salud relacionados con eventos adversos como lesión musculoesquelética, fatiga muscular y complicaciones cardiovasculares, ya que el desarrollo de tales prácticas debe realizarse de manera única, satisfaciendo la demanda individual de cada persona.

Palabras clave: Ejercicio físico; Salud del anciano; Envejecimiento saludable.

\section{Introdução}

O envelhecimento da população está em acentuado crescimento. Mesmo correspondendo a um processo natural do ser humano, nem todas as pessoas gozam do envelhecimento saudável, países desenvolvidos apresentam melhores indicadores comparados aos em desenvolvimento, contudo o interesse por ter vidas mais longas, inerentemente relacionado à busca pela saúde, é uma realidade da sociedade atual (OPAS, 2020).

Com a tendência do aumento no ritmo de envelhecimento, faz-se necessário o desenvolvimento de práticas promotoras de saúde nessa área, com intuito de garantir aos idosos mais anos com qualidade (Miranda et al., 2018; Brasil, 2013).

A prática de exercício físico mostra-se como uma ferramenta para alcance de uma maior longevidade, ou seja, maior tempo de vida, uma vez que esses movimentos programados além de gerar gasto calórico, contribuem para redução da ocorrência de doenças, atuam nas dimensões físicas e cognitivas, envelhecimento saudável e incide na qualidade de vida (Almeida et al., 2020; Lima; Cardoso, 2019; Sousa et al., 2018; Pillatt et al., 2017). 
Para Adamo et al. (2017), a qualidade de vida dos idosos tangencia a preservação da capacidade funcional e da autonomia. E para a Organização Mundial da Saúde a qualidade de vida refere-se à percepção de um indivíduo com sua integração no contexto o qual está inserido, relacionada aos seus objetivos, expectativas, padrões e preocupações (WHO, 1997).

Desse modo, o presente estudo apresenta como objetivo descrever a importância do exercício físico no envelhecimento ativo, a partir de uma reflexão teórica.

\section{Metodologia}

O presente estudo trata-se de um ensaio teórico de caráter reflexivo da literatura, por meio de uma revisão bibliográfica não sistemática, de abordagem qualitativa, sobre o tema "exercício físico e saúde da pessoa idosa", e também através da percepção dos autores acerca da temática.

A pesquisa bibliográfica permite analisar uma temática sob diversos estudos de maneira ampla e concisa (Gil, 2017). Já a abordagem qualitativa, segundo Minayo (2001, p. 21-22), responde a questões muito particulares. Ela se preocupa, nas ciências sociais, com um nível de realidade que não pode ser quantificado. Ou seja, ela trabalha com o universo de significados, motivos, aspirações, crenças, valores e atitudes, o que corresponde a um espaço mais profundo das relações, dos processos e dos fenômenos que não podem ser reduzidos à operacionalização de variáveis.

Como estratégia de busca foram definidas bases de dados eletrônicas, bibliotecas virtuais, respectivamente a Literatura Latino-Americana e do Caribe em Ciências da Saúde (LILACS), Medical Literature Analysis and Retrieval System Online (MEDLINE) e Scientific Eletronic Library Online (SCIELO), utilizando os descritores padronizados pelo Descritores em Ciências da Saúde (Decs): Exercício Físico; Saúde do Idoso; Envelhecimento Saudável, e seus correspondentes em inglês. O levantamento limitou-se aos artigos publicados em português, inglês e espanhol, publicados no período de 2010 a 2020 , disponíveis na íntegra e cujos resultados abrangem o tema desta pesquisa.

As impressões e considerações da análise da literatura serão dispostas em três subtemas "Nuances do envelhecimento"; "Envelhecimento ativo: caminhos para viver bem"; e "Exercício físico na terceira idade"

\section{Resultados e Discussão}

\section{Nuances do envelhecimento}

De acordo com a Organização Pan-Americana de Saúde (OPAS), o envelhecimento é uma parte inerente do ciclo de vida do ser humano, o qual é definido a partir de sucessivas alterações fisiológicas no organismo, que acarreta a redução de tais funções (Brasil, 2006). Segundo Faller et al. (2015), o conceito relaciona-se também com aspectos cronológicos, físicos, biológicos, comportamentais, socioculturais e somam-se a esses fatores as relações com o meio e as experiências de vida de cada indivíduo.

Diante do supracitado, o conhecimento acerca do envelhecimento torna-se imprescindível para adoção de medidas assistenciais ao público idoso. Em 2003 foi instituído o Estatuto do Idoso, destinado a regular os direitos assegurados às pessoas com idade igual ou superior a 60 (sessenta) anos. Além disso, em 2006 foi aprovada a Política Nacional de Saúde da Pessoa Idosa, por meio da portaria $\mathrm{n}^{\text {o }} 2.528$ de 19 de outubro de 2006, com intuito de restaurar, manter e promover a autonomia e independência dos idosos, e para tanto, de acordo com os princípios e diretrizes do Sistema Único de Saúde, são adotadas medidas de caráter coletivo e pessoal, política essa dirigida a todo cidadão brasileiro com mais de 60 anos (Brasil, 2006; Brasil, 2003).

Conforme dados do Instituto Brasileiro de Geografia e Estatística (IBGE), em 2017, o número de idosos ultrapassou a marca de 30 milhões de pessoas idosas. Neste contexto, segundo Miranda et al. (2016), é notório o crescimento da população 
idosa na sociedade, o que demonstra a necessidade de um olhar integral para o processo de envelhecimento com qualidade. Para atingir o envelhecimento saudável é necessário focar na longevidade a longo prazo, o que requer uma reorganização e planejamento no país, enfatizando os serviços de atenção idoso (Miranda et al., 2016).

\section{Envelhecimento ativo: caminhos para viver bem}

As medidas para obter um bom envelhecimento pautam-se na atuação do idoso no ambiente, de forma ativa, no qual esse está inserido. De acordo com a OMS (2009), o envelhecimento ativo configura-se como um processo que contribui para essa atuação, uma vez que proporciona condições de saúde, participação e segurança, de modo a melhorar a qualidade de vida à medida que as pessoas envelhecem. Sousa et al. (2018), revelam que o envelhecimento ativo está ligado às dimensões sociais e também na prática de atividade física de lazer. E para Pillatt et al. (2017), a prática de exercícios físicos beneficia o idoso nas dimensões físicas, cognitivas e de qualidade de vida.

Em consonância ao que foi citado, segundo a Agência Câmara de Notícias, a Política Nacional de Promoção ao Envelhecimento Ativo, projeto de lei 7347/17 estabelece medidas para promover o envelhecimento ativo. Para tanto, as instituições públicas devem desenvolver planos e medidas de prevenção de doenças e estabelecer serviços médicos alternativos para o envelhecimento. O texto também revisou o "Estatuto do Idoso" para determinar que o Estado deve proteger a vida e a saúde dos idosos por meio da implementação de políticas sociais públicas que possibilitem um envelhecimento saudável, ativo e digno. Além disso, o Sistema Único de Saúde também deve oferecer atendimento integral aos idosos (ACN, 2019).

Vale ressaltar que os efeitos positivos do exercício físico no envelhecimento refletem na redução do declínio da saúde e de sua função. Assim, a promoção da prática de exercícios físicos deve ser a pauta central de uma política pública de envelhecimento saudável para prevenção de incapacidades e atenuação de danos na saúde (Moreno-Agostinho et al., 2020).

\section{Exercício físico na terceira idade}

Os benéficos da prática de exercício físico são notórios para a comunidade científica e para adeptos de qualquer modalidade de atividade física. Sabe-se que a prática de atividade física é essencial para promoção da qualidade de vida no envelhecimento (Matsudo et al., 2001).

Neste contexto, Costa et al. (2017) refere que o exercício físico é qualquer atividade física que mantém ou aumenta a aptidão física em geral e tem o objetivo de alcançar a saúde e também a recreação, promovendo o aumento da força, da coordenação e do equilíbrio.

Para Maia et al. (2012), a prática de atividade física é capaz de proporcionar o restabelecimento da saúde, mas também é essencial para promoção da qualidade de vida no envelhecimento. Além disso, segundo Bortoluzzi et al. (2019), os idosos que praticam exercícios físicos também melhoram suas condições de bem-estar (Bortoluzzi et al. 2019; Maia et al., 2012).

A Organização Mundial da Saúde defende que os benefícios da prática da atividade física para a saúde (180 a 300 minutos por semana e 150 a 300 minutos por semana, respectivamente para o sexo masculino e feminino) é notório, visto que contribui significativamente para melhora dos sistemas cardiovascular, metabólico e imunológico, e também melhora o equilíbrio e atua na prevenção de quedas. Outrossim, achados na literatura revelam que níveis altos de prática de exercícios físicos aumentam as chances de viver por mais de 10 anos sem comorbidades crônicas, danos cognitivos e falta de capacidade funcional (Thandi et al. 2018; WHO, 2010).

Cabe mencionar que o número de adeptos ao exercício físico é maior para mulheres, comparando o quantitativo de homens que se exercitam. Embora seja necessário refletir que o contexto histórico feminino demonstra que as mulheres 
exercem funções no cotidiano que dificultam a inserção do exercício físico no seu estilo de vida, como o somatório da jornada de trabalho e o cuidar do lar (Ferretti et al., 2020).

\section{Considerações Finais}

Ao longo do estudo foi possível conhecer as inúmeras vantagens da prática de exercício físico para a saúde do idoso, como a melhora nas capacidades cognitivas e funcionais, redução no risco de doenças crônicas e incapacitantes, aumento da qualidade de vida e bem-estar, além de ser responsável pela inserção do idoso nos ambientes sociais. No entanto, há lacunas quanto à execução segura para cada modalidade de exercício físico e os possíveis riscos à saúde relacionados a eventos adversos como lesão musculoesquelética, fadiga muscular e complicações cardiovasculares, uma vez que o desenvolvimento de tais práticas deve ser realizados de forma singular, atendendo a demanda individual de cada pessoa. Deste modo, o ensaio traz a reflexão para o olhar holístico e humanizado na implementação das atividades físicas no envelhecimento, almejando fomentar a literatura e colaborar com a difusão da importância do exercício físico como aliado para a manutenção da saúde e qualidade de vida.

Espera-se que o presente estudo possa contribuir para o desenvolvimento de ações para promoção da saúde da pessoa idosa, bem como ampliar o conhecimento do tema, a fim de difundir a importância da prática de exercício físico na busca pelo envelhecimento saudável.

Ademais, mostra-se relevante para abordagens futuras analisar, a partir da fala das pessoas idosas, de que forma a prática do exercício físico impacta na qualidade de vida, observando se estes são acompanhados por profissionais habilitados ou se o exercício físico é realizado de forma autônoma e independente.

\section{Referências}

Adamo, C. E. E.; Bastos, M. T.; Cantarelli, G. C. F.; Sousa, I. F.; Almeida, R. J. (2017). Universidade aberta para a terceira idade: o impacto da educação continuada na qualidade de vida dos idosos. Revista Brasileira de Geriatria e Gerontologia, 20(4), 545-555. https://dx.doi.org/10.1590/198122562017020.160192 .

Almeida, B. L.; Souza, M. E. B. F.; Rocha, F. C.; Fernandes, T. F.; Evangelista, C. B.; Ribeiro, K. S. M. A. (2021). Quality of life of elderly people who practice physical activities / Qualidade de vida de idosos que praticam atividade física. Revista De Pesquisa Cuidado é Fundamental Online, 12 , $432-436$. https://doi.org/10.9789/2175-5361.rpcfo.v12.8451.

Bortoluzzi, E. C.; Pancotte, J.; Doring, M.; Graeff, D. B.; Alves, A. S.; Portella, M. R.; Scortegagna, H. M.; Dalmolin, B. M. (2018). Autopercepção de saúde de idosas praticantes de atividades físicas e fatores associados. Estudos interdisciplinares sobre o envelhecimento, 23, 2. https://doi.org/10.22456/23162171.64619 .

Brasil. (2013). Ministério da Saúde, Secretaria de Atenção à Saúde. Estatuto do idoso [Internet]. Brasília: Ministério da Saúde, 70. http://bvsms.saude.gov.br/bvs/publicacoes/estatuto_idoso_3edicao.pdf.

Brasil. (2006). Ministério da Saúde. Secretaria de Atenção à Saúde. Departamento de Atenção Básica. Envelhecimento e saúde da pessoa idosa. Brasília: Ministério da Saúde, 192. https://bvsms.saude.gov.br/bvs/publicacoes/evelhecimento_saude_pessoa_idosa.pdf

Câmara dos deputados. (2019). Comissão aprova Política Nacional de Promoção ao Envelhecimento Ativo. Política e Administração Pública. Agência Câmara de Notícias. https://www.camara.leg.br/noticias/597568-comissao-aprova-politica-nacional-de-promocao-ao-envelheciment.

Costa, F. S.; Cabral, C. O. M.; Rodrigues, J. P.; Santos, A. M. (2017). Idosos e Exercícios Físicos: Motivações e Contribuições para Saúde e o Lazer. LICERE - Revista Do Programa De Pós-graduação Interdisciplinar Em Estudos Do Lazer, 20(4), 212-237. https://doi.org/10.35699/1981-3171.2017.1732.

Faller, J. W.; Teston, E. F; Marcon, S. S. (2015). A velhice na percepção de idosos de diferentes nacionalidades. Texto \& Contexto - Enfermagem, 24(1), 128137. https://doi.org/10.1590/0104-07072015002170013.

Ferretti, F.; Macagnan, D.; Canei, F. C.; Silva, M. R.; Santos, M. P. M. (2020). Physical activity level among older adultsover 70 years old and very old adults. Fisioterapia em Movimento, 33, e003327. Epub April 17, 2020. https://doi.org/10.1590/1980-5918.033.ao27.

Gil, A. C. Como elaborar projetos de pesquisa. 6. ed. São Paulo: Atlas, 2017.

IBGE. (2018). Número de idosos cresce 18\% em 5 anos e ultrapassa 30 milhões em 2017. Instituto Brasileiro de Geografia e Estatística. Agência IBGE Notícias, 2018. https://agenciadenoticias.ibge.gov.br/agencia-noticias/2012-agencia-de-noticias/noticias/20980-numero-de-idosos-cresce-18-em-5-anos-eultrapassa-30-milhoes-em-2017. 
Lima, A. P.; Cardoso, F. B. (2019). Atividade Física de Lazer em Idosos com Diabetes Tipo 2: Estudo de Base Populacional. LICERE - Revista Do Programa De Pós-graduação Interdisciplinar Em Estudos Do Lazer, 22(2), 1-17. https://doi.org/10.35699/1981-3171.2019.13537.

Maia, M. F. M.; Ruas, A. R. M.; Rocha, F. P.; Oliveira, N. F. R.; Souza, J. S. (2012). A autoestima em uma amostra de idosos com prática de caminhada orientada. Motricidade, 8(2),1085-1088.[acesso 4 de Maia de 2021]. ISSN: 1646-107X. https://www.redalyc.org/articulo.oa?id=273023568138.

Matsudo, S. M.; Matsudo, V. K. R.; Neto, T. L. B. (2001). Atividade física e envelhecimento: aspectos epidemiológicos. Revista Brasileira de Medicina do Esporte, 7(1), 2-13. https://dx.doi.org/10.1590/S1517-86922001000100002.

Measuring Quality of Life. (1997). The World Health Organization Quality of Life Instruments. Programme on Mental Health. Division of mental health and prevention of substance abuse World Health Organization, 1-12. https://www.who.int/mental_health/media/68.pdf.

Minayo, M. C. S., (2001). Pesquisa Social. Teoria, método e criatividade. 18 ed. Petrópolis.

Miranda, G. M. D.; Mendes, A. C. G.; Silva, A. L. A. (2016). O envelhecimento populacional brasileiro: desafios e consequências sociais atuais e futuras. Revista Brasileira de Geriatria e Gerontologia, 19(3), 507-519. https://doi.org/10.1590/1809-98232016019.150140.

Moreno-Agostino, D.; Daskalopoulou, C.; Wu, Y.-T., Koukounari, A.; Haro, J. M.; Tyrovolas, S.; Panagiotakos, D. B., Prince, M.; Prina, A. M. (2020). The impact of physical activity on healthy ageing trajectories: Evidence from eight cohort studies. The International Journal of Behavioral Nutrition and Physical Activity, 17, 92. https://doi.org/10.1186/s12966-020-00995-8.

OMS. (2009). Guia Global das Cidades Amigas das Pessoas Idosas. Fundação Calouste Gulbenkian, $2009 . \quad 76$ p. https://apps.who.int/iris/bitstream/handle/10665/43755/9789899556867_por.pdf?sequence=3.

Pillatt, A. P.; Nielsson, J.; Schneider, R. H. (2019). Efeitos do exercício físico em idosos fragilizados: uma revisão sistemática. Fisioterapia e Pesquisa, 26(2), 210-217. Epub July 18, 2019. https://doi.org/10.1590/1809-2950/18004826022019.

Thandi, M. K. G.; Phinney, A.; Oliffe, J. L.; Wong, S.; McKay, H.; Sims-Gould, J.; Sahota, S. (2018). Engaging Older Men in Physical Activity: Implications for Health Promotion Practice. American journal of men's health, 12(6), 2064-2075. https://doi.org/10.1177/1557988318792158.

Sousa, N. F. S.; Lima, M. G.; Cesar, C. L. G.; Barros, M. B. A. (2018). Envelhecimento ativo: prevalência e diferenças de gênero e idade em estudo de base populacional. Cadernos de Saúde Pública, 34(11), e00173317. Epub November 23, 2018.https://doi.org/10.1590/0102-311x00173317.

WHO. (2010). Global recommendations on physical activity for health. World Health Organization. Geneva. https://https://www.who.int/dietphysicalactivity/global-PA-recs-2010.pdf. 